\title{
Effet de la crise de Covid sur la persistance et l'asymétrie de la volatilité du marché boursier marocain
}

\author{
Mohamed NABOUK \\ Département de gestion, Faculté d'économie et gestion. Hassan $1^{\text {er }}$, Maroc
}

\begin{abstract}
Résumé : La volatilité a été toujours la matière première des activités du marché boursier dont il faut toujours appréhender, analyser et prévoir. Son évolution dépend à la fois des facteurs macro-économiques (inflation, taux de chômage, etc...) et des facteurs spécifiques (résultats des entreprises cotées). Mais parfois des événements improbables : par exemple crise des sub-primes 2008 ou récemment la pandémie de Covid créent des turbulences et des incertitudes et qui conduisent le niveau de la volatilité à des pics très élevés suite aux fluctuations des valorisations des actifs financiers cotés à la bourse. Cette pandémie, et qui a affecté l'ensemble des marchés mondiaux, n'a pas épargné la bourse des valeurs de Casablanca. L'objet de ce papier est d'une part d'analyser l'impact de cette pandémie sur le comportement de la volatilité et son degré de persistance malgré l'optimisme apporté par les nouveaux vaccins et d'autres de voir dans quelle mesure la crise a créé ou amplifié l'effet d'asymétrie suite aux chocs dus aux événements négatifs. Les conclusions de ce travail ont abouti d'une part à la confirmation de l'augmentation de la persistance de la volatilité pour l'ensemble du marché et d'autres part à l'apparition d'un effet d'asymétrie dans le comportement de la volatilité.
\end{abstract}

Mots-clés : volatilité conditionnelle, Modélisation GARCH, persistance de la volatilité, effet d'asymétrie des chocs negatifs.

\section{INTRODUCTION}

La volatilité, conséquence des fluctuations des cours, est essentiel pour les investisseurs de la bourse. C'est la matière première des activités de marché et qui assure une fonction de contrepartie pour couvrir les risques de l'économie réelle. Donc chercher à la réduire à néant ça serait inutile. D'une part puisque le portefeuille marché est caractérisé toujours par un risque à la foi systématique et spécifique dont l'ampleur dépend des anticipations macro et micro économique en tenant parfois des facteurs géopolitique. D'autres part, sans la volatilité pas d'évolution de cours donc pas de gain possible.

La question fondamentale c'est que le marché doit trouver un juste équilibre, autrement dit, on ne peut pas accepter ni une volatilité excessive à la hausse ou à la baisse des cours, ni une volatilité très faible pour préserver l'attractivité des places boursières. Ce pendant plusieurs études empiriques ont démontré certains faits stylisés qui caractérisent le comportement de la volatilité : volatilité excessive des marchés qui perturbent 
la formation du prix des actifs financiers (Shiller ${ }^{1}$ ), la non de stabilité ou l'absence de l'homoscédasticité de la volatilité dans le temps et son caractère prévisible de plus en plus et enfin l'effet de levier ou d'asymétrie et la persistance à court terme et à long terme de la volatilité. ,

Les modèles de type $\mathrm{ARCH}$ ou GARCH qui prennent en considération ces propriétés sont les modèles à hétéroscédasticité conditionnelle. La modélisation de la volatilité dite conditionnelle à partir de ces modèles permet à la foi l'analyse de la persistance et la prévision de la volatilité. Les implications de cette modélisation est très utile en matière d'allocation des actifs pour les gestionnaires de portefeuille du fait que les hypothèses de la théorie moderne de gestion portefeuille ne sont plus vérifiées particulièrement la normalité de la distribution des séries des rendements.

L'instabilité permanente de la volatilité a été certainement amplifiée par la pandémie de Covid-19. Cette crise sans précédent, aussi bien par sa nature que par l'ampleur de ses conséquences, a frappé une large partie du monde et mis à mal l'économie mondiale. Les marchés de capitaux ont enregistré une volatilité très élevée et des baisses importantes des prix des actifs financiers, rendant nécessaire l'adoption par les autorités publiques et les régulateurs des marchés financiers de mesures adaptées à cette situation.

L'objet de ce papier est d'analyser le comportement de la volatilité du marché boursier marocain, avant et après la pandémie de Covid-19 en admettant une approche comparative entre les principaux indices de la place de Casablanca : MASI, FTSE 15 et FTSE ALL. Cette analyse nous permettra de voir dans quelle mesure la crise de Covid a produit un choc de volatilité à court ou à long terme et à amplifier l'effet d'asymétrie en procédant à une analyse comparative entre les trois indices MASI, FTSE 15 et FSTE ALL.

\section{REVUE DE LITTERATURE SUR LES MODELES DE LA VOLATILITE ET EFFET D'ASYMETRIE.}

\section{1. Le modèle standard GARH}

Le modèle GARCH dans sa forme générique propose une estimation intuitive de la volatilité, cette dernière étant la somme des rendements passés pondérés. La version généralisée GARCH (p, q), qui est la plus exploitée, est décrite par deux équations.

- La première donne le rendement moyen d'un actif ou d'un indice financier :

$$
R_{t}=\mu+\varepsilon_{t}
$$

\footnotetext{
${ }^{1}$ R. Shiller, « Do Stock Prices Move too Much to be Justifi ed by Subsequent Changes in Dividends », American Economic Review, 71, 3, 1981, p. 421-436.
} 
ou $\mu$ représente la valeur moyenne des rendements et $\varepsilon_{t}$ est l'innovation, c'est-à-dire, qui distingue le rendement journalier de sa valeur moyenne en incorporant les dernières nouvelles. Cette innovation est modélisée par une variable aléatoire gaussienne d'écart-type $\sigma_{\mathrm{t}}$.

- La deuxième équation fait dépendre la volatilité des rendements et des volatilités passées. Pour un modèle GARCH d'ordre (p, q) :

$$
\sigma_{t}^{2}=\omega+\sum_{i=1}^{p} \alpha_{i} \varepsilon_{t-i}^{2}+\sum_{j=1}^{q} \beta_{j} \sigma_{t-j}^{2}
$$

Dans cette formule, $\mathrm{p}$ et q donnent les plages temporelles passées qui seront pris en compte pour déterminer la volatilité présente.

$\omega$ : La variance inconditionnelle.

$\alpha$ : est un paramètre qui gouverne l'impactdu choc passé.

$\beta$ : est un paramètre qui s'interprète comme la vitesse du retour à la volatilité minimale $\omega$.

La spécification la plus répandue est le GARCH $(1,1)$ permettant une représentation assez générale des processus de volatilité conditionnelle. Dans sa forme la plus simple le GARCH $(1,1)$ s'écrit comme suit :

$$
\sigma_{t}^{2}=\omega+\alpha \varepsilon_{t-1}^{2}+\beta \sigma_{t-1}^{2}
$$

On note, $\omega=\gamma V l$, le modèle $s^{\prime}$ écritcomme suit :

$$
\sigma_{t}^{2}=\gamma V_{L}+\alpha \varepsilon_{t-1}^{2}+\beta \sigma_{t-1}^{2}
$$

$\boldsymbol{\gamma}, \boldsymbol{\alpha}$ et $\boldsymbol{\beta}$ représentent le poids attribués respectivement à $\mathrm{V}_{\mathrm{L}}$ (volatilité non conditionnelle ou volatilité à long terme); $\varepsilon^{2}$ t-1 et $\sigma^{2}$ t-1 et la somme des coefficients de pondérations est égale à $1(\mathrm{\gamma}+\alpha+\beta=1)$.

Cette formulation est généralement utilisée pour estimer les paramètres $\omega ; \alpha$ et $\beta$. De cette estimation on peut calculer $\gamma$ qui vaut $\gamma=1-\alpha-\beta$ et la variance à long terme $V_{L}=\omega / \gamma$.

La stabilité du processus GARCH est obtenu en imposant $\alpha+\beta<1$. Si non le coefficient de pondération appliqué à la variance à long terme sera négatif.

Le modèle GARCH est une solution capable de reproduire deux faits stylisés des séries financières à savoir :

- $\quad$ Le Clustering ou le regroupement de la volatilité du rendement par grappe comme qu'il a été démontré par B. Mandelbort. Les rendements petits en taille sont suivis par des rendements petits et les rendements grands en taille sont suivis par des rendements grands avec des périodes plus calmes.

- La non-normalité de la distribution des rendements. Cette distribution est souvent léptokurtiques cela signifie que la probabilité des rendements des rendements extrêmes positifs ou négatifs est plus grande par 
rapport à celle d'une loi normale. Les séries chronologiques financières présentent généralement des queues épaisses qu'on est qualifié d'excès de kurtosis. Ainsi le coefficient du kurtosis (le quatrième moment central normalisé par le carré de la variance) est généralement supérieur au kurtosis d'une variable aléatoire gaussienne.

A l'origine, Le modèle GARCH est une extension du modèle ARCH. Pour ce dernier le $\beta=0$.

Le modèle s'écrit comme suit :

- Pour un ARCH d'ordre $1: \boldsymbol{\sigma}_{t}^{2}=\boldsymbol{\omega}+\boldsymbol{\alpha} \boldsymbol{\varepsilon}^{2}{ }_{t-1}$

- Pour un ARCH d'ordre p : $\sigma_{t}^{2}=\omega+\sum_{i=1}^{p} \alpha_{i} \varepsilon_{t-i}^{2}$

Cette forme standard et généralise du modèle GARCH $(1,1)$ communément utilisés dans la modélisation des séries financières, impliquent une persistance des chocs de volatilité respectivement faible et infinie et en plus elle est incapable de reproduire les effets d'asymétrie.

A partir de la modélisation GARCH on peut apprécier la persistance de la volatilité. Elle est mesurée par la somme des coefficients $\boldsymbol{\alpha}$ et $\boldsymbol{\beta}$. Plus que la somme tend vers 1 ou l'unité, sans qu'elle soit supérieure pour garantir la stabilité du modèle, plus la volatilité est persistante.

Cette persistance de la volatilité, et compte tenu du principe de la modélisation GARCH, peut être prévisible avec un effet de retour vers la volatilité non conditionnelle $\left(V_{L}\right)$.

$$
\begin{aligned}
& \sigma_{t}^{2}={ }_{V} V_{L}+\alpha \varepsilon_{t-1}^{2}+\beta \sigma_{t-1}^{2} \\
& \sigma_{t}^{2}=(1-\alpha-\beta) V_{L}+\alpha \varepsilon_{t-1}^{2}+\beta \sigma_{t-1}^{2} \\
& \sigma_{t}^{2}-V_{L}=(1-\alpha-\beta) V_{L}+\alpha \varepsilon_{t-1}^{2}+\beta \sigma_{t-1}^{2} \\
& \sigma_{t}^{2}-V_{L}=\alpha\left(\varepsilon_{t-1}^{2}-V_{L}\right)+\beta\left(\sigma_{t-1}^{2} V_{L}\right) \\
& E\left(\sigma_{n+t}^{2}-V_{L}=\alpha\left(\varepsilon_{t-1}^{2}-V_{L}\right)+\beta\left(\sigma_{t-1}^{2} V_{L}\right)\right.
\end{aligned}
$$

On peut estimer $\mathrm{E}\left(\varepsilon_{\mathrm{t}-1}^{2}\right)$ par $\sigma_{\mathrm{t}-1}^{2}$

$$
E\left(\sigma_{t+1}^{2} l-V_{L}\right)=(\alpha+\beta)\left(\sigma_{t-1}^{2}-V_{L}\right)
$$

L'utilisation répétée de cette équation nous conduit à :

$$
\begin{aligned}
& E\left(\sigma^{2}{ }_{t+n}-V_{L}\right)=(\boldsymbol{\alpha}+\beta)^{n}\left(\sigma^{2}-V_{L}\right) \\
& E\left(\sigma^{2}{ }_{t+n}\right)=V_{L}+(\boldsymbol{\alpha}+\beta)^{n}\left(\sigma^{2}{ }_{t}-V_{L}\right)(1)
\end{aligned}
$$

L'équation (1) nous donne une prévision de la variance conditionnelle pour la période $(\boldsymbol{t}+\boldsymbol{n})$ à partir des seules informations disponibles à la fin de la journée (n-1). Comme on peut remarquer dans l'équation (1), quand $\boldsymbol{\alpha}+\beta<1$, le dernier terme de l'équation diminue à mesure que (n) augmente. Plus nous nous projetons dans le futur et plus les prévisions de la volatilité conditionnelle tendent vers $V_{L}$ (variance à long terme). 
Dans la plupart des études empiriques (Engle et Bollertilev, 1986, French, Schwert et Starnbaugh, 1987, Chou, 1988, Baillie et DeGennaro, 1990, Carnpbell et Hent- tichel, 1992), on accepte l'hypothèse d'une forte persistance de volatilité en haute fréquence. En revanche, la persistance tend à décroître sensiblement lorsque la fréquence des données diminue, ceci fournit une indication sur la stationnarité de la variance conditionnelle (Poon et Taylor, 1992). Cette étude étant réalisée avec des données quotidiennes, des phénomènes de persistance de la volatilité peuvent donc être observés.

\section{2-2 La volatilité conditionnelle et asymétrie}

Dans leur version initiale, les modèles ARCH et GARCH ne permettent pas de tenir compte de l'hypothèse d'asymétrie. Pour corriger ce "défaut", des travaux plus: récents ont proposé des extensions de cette approche de façon à tenir en compte de ces effets. Plusieurs formulations ont ainsi été proposées (Nelson, 1991, Engle et $\mathrm{Ng}$, 1993, Glosten, Jagannathan et Runkle, 1993, 1993, Zakoïan, 1991, et Hentschel, 1995).

L'Effet de levier d'asymétrie distingue entre l'effet des valeurs passées négatives et l'effet des valeurs passées positives sur la volatilité des cours ou de rendements. Les baisses de cours tendent à engendrer une augmentation de la volatilité supérieure à celle induite par une hausse des cours de même ampleur.

Le modèle GARCH est une réponse de la volatilité future aux variations des prix déterminées uniquement par l'ampleur de ces dernières et non par leur signe. Par contre, les modèles GARCH asymétriques étendent ces spécifications en incorporant de l'asymétrie dans la réponse de la volatilité aux variations de prix.

Trois spécifications peuvent être particulièrement retenus.

\section{2-2-1. Le modèle EGARCH}

Il s'agit du modèle EGARCH ou GARCH exponentiel qui a été développé par Nelson (1991). Dans sa forme la plus simple le EGARCH $(1,1)$ s'écrit comme suit :

$$
\ln \left(\sigma_{t}\right)=\alpha_{0}+\alpha_{1}\left(\left|\varepsilon_{t-1}\right|-E\left(\varepsilon_{t-1}\right)\right)+\gamma \varepsilon_{t-1}+\beta \ln \left(\sigma_{t-1}\right)
$$

Cette formulation présente un double intérêt :

- L'écriture porte sur le logarithme de la variance conditionnelle $\sigma_{t}^{2}$. Par conséquant aucune restriction n'est imposée sur les différents paramètres de l'équation pour assurer la positivité de $\sigma_{t}^{2}$. Notez que le côté gauche de l'équation donne la variance conditionnelle en logarithme. Cela implique que l'effet de force de levier est plutôt exponentiel que quadratique, et l'on garantit que les prévisions de la variance conditionnelle ne sont pas négatives. La présence d'effets de force de levier peut être évaluée selon l'hypothèse que $\gamma=0$ ou non. L'impact est asymétrique si $\gamma \neq 0$ et dans le cas contraire, l'effet est symétrique, c'est-à-dire, les variations des prix positives ou négatives produisent les mêmes amplitudes de volatilité. 
- La variance conditionnelle $\sigma_{t}^{2}$ fait apparaître un effet de signe, correspondant $\grave{a} \gamma \varepsilon_{t-1}$ un effet d'amplitude mesuré par $\alpha_{1}\left(\left|\varepsilon_{t-1}\right|-\mathrm{E}(\varepsilon)\right)$.

Le coefficient $\boldsymbol{\alpha}_{\mathbf{1}}+\boldsymbol{\gamma}$ permet de mesurer l'effet total d'un choc positif sur la volatilité et pour un choc négatif, l'impact total correspond au coefficient $\boldsymbol{\alpha}_{\mathbf{1}}-\boldsymbol{\gamma}$.

En utilisant le modèle EGARCH, Black trouve que la volatilité sur le marché boursier a tendance à augmente après les rentabilités négatives et a tendance à baisser après les rentabilités positives. Le modèle EGARCH exploite cette régularité empirique en mettant la variance conditionnelle en fonction de la taille et le signe de résidus retardés. Etant différent par rapport au modèle GARCH (p, q).

\section{2-2-2. Le modèle GJR (Glosten, Jagannathan et Runkel, 1993)}

Le modèle GJR est défini par :

$$
\sigma^{2}=\alpha_{0}+\alpha_{1} \varepsilon_{t-1}^{2}+\gamma \vartheta_{t-1} \varepsilon_{t-1}^{2}+\beta\left(\sigma_{t-1}^{2}\right)
$$

Avec $\boldsymbol{\vartheta}_{\mathrm{t}-1}$ égale à 1 si $\varepsilon_{\mathrm{t}-1}<0$, et 0 pour les autres cas . Pour assurer la positivité de la volatilité, il faut que :

$$
\alpha_{0}>0 ; \alpha_{1} \geq 0 ; \alpha_{1}+\gamma \geq 0 \text { et } \beta \geq 0 \text {. }
$$

Le modèle est stable à condition : $\boldsymbol{\alpha}_{\mathbf{1}}+\boldsymbol{\beta}+\boldsymbol{\gamma} / \mathbf{2}<\mathbf{1}$ est vérifiée ${ }^{2}$.

Le coefficient $\boldsymbol{\gamma}$ permet particulièrement de mesurer l'effet complémentaire d'un choc négatif sur la volatilité sachant que l'impact total correspond à la somme de $\boldsymbol{\alpha}_{\mathbf{1}}+\boldsymbol{\gamma}$. Dans le cas d'un choc positif, l'impact total correspond au coefficient $\boldsymbol{\alpha}_{\mathbf{1}}$.

\section{2-2-3. Le modèle TGARCH (Threshold GARCH, Zakoïan , 1991)}

La formulation du modèle s'écrit comme suit :

$$
\sigma_{\mathrm{t}}=\alpha_{0}+\alpha_{1}\left|\varepsilon_{\mathrm{t}-1}\right|+\gamma \boldsymbol{\vartheta}_{\mathrm{t}-1}\left|\varepsilon_{\mathrm{t}-1}\right|+\beta \sigma_{\mathrm{t}-1}
$$

Avec $\boldsymbol{\vartheta}_{\mathbf{t}-\mathbf{1}}$ égale à 1 si $\varepsilon_{\mathrm{t}-1}<0$, et 0 pour les cas contraire.

Pour assurer la positivité de la volatilité, il faut : $\alpha_{0}>0 ; \alpha_{1} \geq 0 ; \alpha_{1}+\gamma \geq 0$ et $\beta \geq 0$.

Le modèle TGARCH permet la même interprétation des coefficients du modèle dans le cas d'un choc négatif ou un choc positif. La différence fondamentale entre les deux modèles réside dans sa formulation. Le GJR est une expression de la variance conditionnelle et le TGARCH est une expression de la volatilité conditionnelle. C'est pourquoi dans les études empiriques le modèle GJR GARCH est préférable à la spécification TGARCH. A l'opposé le EGARCH est expression du logarithme de la volatilité conditionnelle. 


\section{ANALYSE ET MODELISATION DE LA VOLATILITE DU MARCHE}

\section{3-1- Observation empirique de la volatilité réalisée du marché boursier marocain}

Avant d'analyser la volatilité du marché boursier marocain, il est nécessaire d'étudier les caractéristiques des rendements journaliers des indices à étudier.

Les caractéristiques statistiques descriptives des rendements journaliers des 3 indices sont réalisés déterminées pour la période s'étalant entre le $\underline{\mathbf{5 / 7 / 2 0 1 0 - 1 0 / 0 5 / 2 0 2 1}}$. Elles sont résumées dans le tableau suivant :

Tableau 1 : caractéristiques statistiques des rendements quotidiens

\begin{tabular}{|l|l|l|l|}
\hline indice & FTSE ALL & MASI & FSTE 15 \\
\hline Moyenne & $-0,00044 \%$ & $0,00084 \%$ & $0,00163 \%$ \\
\hline Médiane & 0.000000 & $4.00 \mathrm{E}-05$ & 0.000000 \\
\hline Maximum & 0.056827 & 0.053054 & 0.066438 \\
\hline Minimum & -0.099990 & -0.092317 & -0.097901 \\
\hline Ecart type & 0.007541 & 0.006811 & 0.007879 \\
\hline Skewness & -1.020928 & -1.207770 & -0.915240 \\
\hline Kurtosis & 22.29636 & 25.24270 & 21.38556 \\
\hline Statistique Jarque-Bera & 42389.73 & 56356.11 & 38433.65 \\
\hline Probabilité & 0.000000 & 0.000000 & 0.000000 \\
\hline
\end{tabular}

D'après le tableau (1), les trois indices présentent un Kurtosis largement supérieur à 3 (3 est le coefficient d'une distribution normale) et un coefficient d'asymétrie négative ce qui signifie qu'il y a plus de rendement extrême négatif que positif. Cette asymétrie peut soupçonner la présence d'un effet d'asymétrie dans l'évolution de la volatilité. Ces caractéristiques observées donnent en fin de compte une distribution différente par rapport à la loi normale ce que confirme la statistique de Jarque-Bera.

L'analyse empirique de la volatilité des principaux indices peut aborder selon deux horizons :

- Un horizon à long terme en calculant la volatilité des rendements logarithmiques sur période de 250 jours (volatilité annuelle ${ }^{3}$ ).

- Un horizon à court terme en calculant la volatilité sur une période de 20 jours (volatilité mensuelle ${ }^{4}$.

$\bar{R}=\frac{1}{n} \sum_{1}^{n} R i ; \bar{R}:$ représente le rendement logarithmique moyen quotidien ou journalier et donc l'écart type $\mathrm{j}$ est égal :

\footnotetext{
${ }^{3}$ Le nombre de séance boursière par an est en moyenne de 250 séances.

${ }^{4}$ Le nombre de séance par mois est de 20 séances, soit 5 séances par semaine.
} 


$$
\sigma j(R)=\sqrt{\frac{1}{n-1}} \sum_{i=1}^{n}(R i-\bar{R})^{2}
$$

A partir de la volatilité quotidienne, on peut calculer la volatilité annuelle :

$\sigma(R)$ annuel $=\sqrt{250 *} \sigma j(R)$

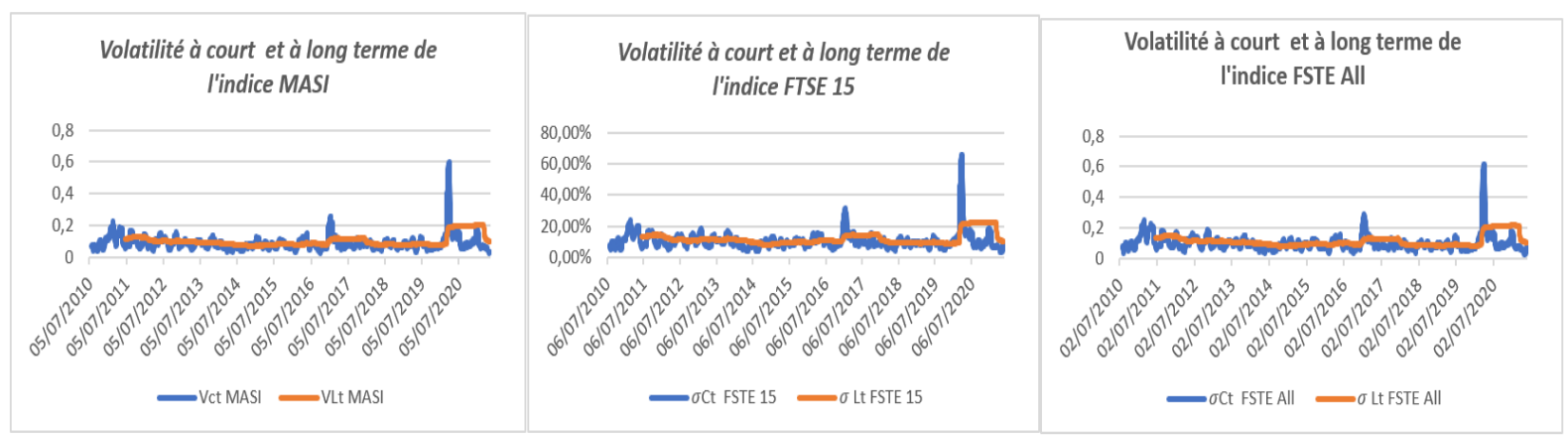

Figure 1 : Evolution de la volatilité historique des indices MASI, FTSE 15 et FTSE ALL Liquid entre 5/7/2010-10/05/2021(Source auteur d'après les données du site de la bourse)

L'examen graphique de la volatilité à court terme et historique révèle :

- L'examen du graphique révèle une forte explosion de la volatilité à court terme pour les 3 indices depuis le début de la période de crise de Covid. Après, suite aux mesures prises par les autorités marocaines, les choses commencent à revenir à la normale.

- La volatilité à court terme pour les 3 indices fluctue fortement par rapport à la volatilité historique avec des fortes amplitudes à la hausse et à la baisse. Ce qui confirme la forte dispersion ou l'instabilité temporelle de la volatilité. Cette instabilité a été accentué fortement depuis le début de la crise de Covid.

- La volatilité à court terme de l'indice MASI est moins forte que celle de l'indice FSTE ALL LIQUID, elle-même inférieure à la volatilité de FSTE 15. Donc on peut dire dans le court terme la liquidité est un facteur important dans l'évolution des indices.

- La volatilité n'est pas constante. Après des périodes de faibles volatilités succèdent des périodes de forte volatilité avec un pic depuis l'installation de la crise de covid. Ceci confirme la persistance de la volatilité avec un effet d'asymétrie puisque pour la période le coefficient de corrélation est négatif 
$(-5 \%)$ entre la variations de l'indice MASI et la volatilité à court terme. Ce coefficient de corrélation était positif de $3 \%$. On déduit que la crise de covid a produit un effet de levier ou d'asymétrie à confirmer.

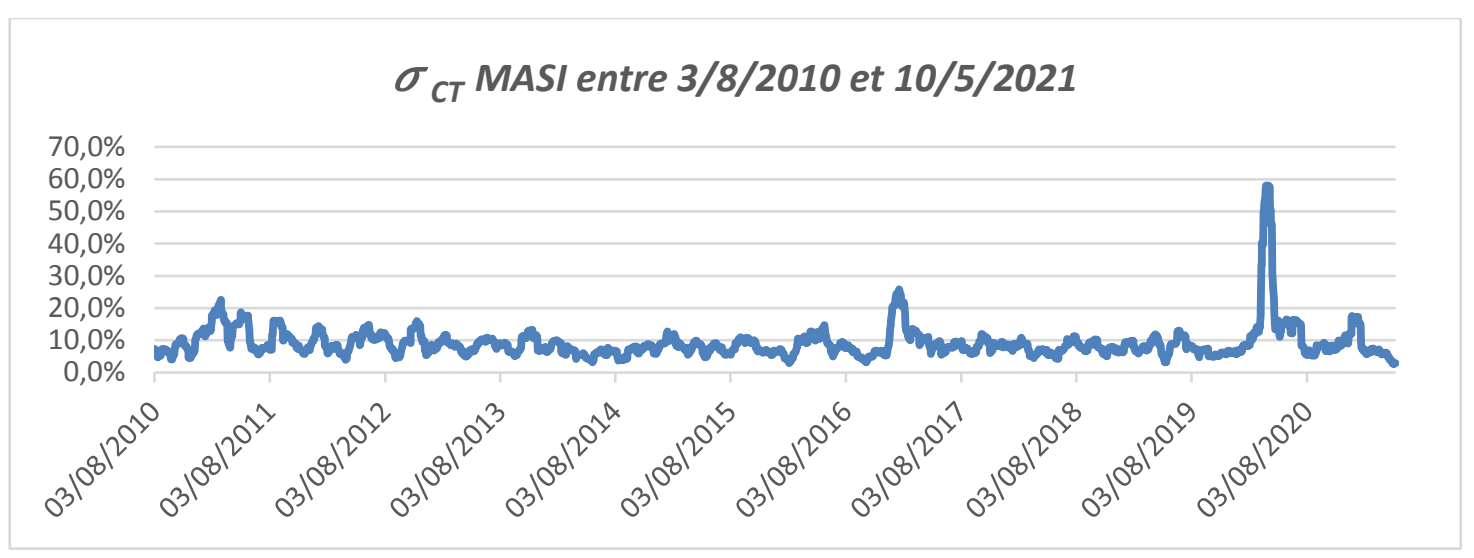

Figure 2 : Evolution de la volatilité à court terme.

Cette instabilité de la volatilité est un handicap majeur pour la gestion des risques de portefeuille par l'approche moyenne-variance ${ }^{5}$.

Tableau 2: MAX MIN et Médiane des 3 indices pour la même période avant et après période le crise de Covid

\begin{tabular}{|c|c|c|c|c|c|c|}
\hline Indice & \multicolumn{2}{|c|}{ MASI } & \multicolumn{2}{|c|}{ FTSE 15} & \multicolumn{2}{|c|}{ FTSE ALL } \\
\hline$\sigma(e ́ c a r t$ type $)$ & $\sigma_{\mathrm{CT}}$ & $\sigma_{\mathrm{LT}}$ & $\sigma_{\mathrm{CT}}$ & $\sigma_{\mathrm{LT}}$ & $\sigma_{\mathrm{CT}}$ & $\sigma_{\mathrm{LT}}$ \\
\hline (le 5/7/2010 au 31/12/2019) & $25,91 \%$ & $12,81 \%$ & $31,64 \%$ & $14,86 \%$ & $28,45 \%$ & $14,92 \%$ \\
\hline Médiane (le 5/7/2010 au 31/12/2019) & $8,00 \%$ & $8,63 \%$ & $9,50 \%$ & $10,59 \%$ & $8,85 \%$ & $9,88 \%$ \\
\hline$(5 / 7 / 2010$ au $10 / 5 / 2021)$ & $58,02 \%$ & $20,4 \%$ & $65,9 \%$ & $22,7 \%$ & $61,9 \%$ & $21,8 \%$ \\
\hline MEDIANE (5/7/2010 au $10 / 5 / 2021)$ & $8,12 \%$ & $8,9 \%$ & $9,6 \%$ & $10,8 \%$ & $8,9 \%$ & $10,1 \%$ \\
\hline
\end{tabular}

Le tableau (2) démontre clairement l'impact de la crise de Covid dans l'augmentation de la volatilité réalisée. Le niveau des valeurs MEDIANE et MAX ont explosés par rapport à la période avant Covid.

\section{3-2 MODELISATION DE LA VOLATILITE : APPLICATION AU MARCHE BOURSIER MAROCAIN}

Cette modélisation devrait répondre à deux questions :

- Comment la persistance de volatilité a été impactée par la crise de covid ?

\footnotetext{
${ }^{5}$ L'approche moyenne-variance est le modèle de base pour l'allocation des portefeuilles et qui a été développé par
} Markowitz 
Mohamed NABOUK : Effet de la crise de Covid sur la persistance et l'asymétrie de la volatilité du marché...

- Est-ce que cette crise a fait apparaitre ou accentuer d'asymétrie ?

\section{3-2-1 Analyse de la persistance de la volatilité pour le modèle GARCH}

La modélisation GARCH $(1,1)$ de la volatilité s'écrit comme suit :

$$
\sigma_{t}^{2}=\omega+\alpha \varepsilon_{t-1}^{2}+\beta \sigma_{t-1}^{2}
$$

On note, $\boldsymbol{\omega}=\gamma \boldsymbol{V} \boldsymbol{l}$, le modèle s'écrit comme suit :

$$
\sigma_{t}^{2}={ }_{\gamma} V_{L}+\alpha \varepsilon_{t-1}^{2}+\beta \sigma_{t-1}^{2}
$$

Cette formulation est généralement utilisée pour estimer les paramètres $\boldsymbol{\omega} ; \boldsymbol{\alpha}$ et $\beta$.

De cette estimation, on peut calculer $\gamma$ et qui vaut $\gamma=1-\boldsymbol{\alpha}-\beta$ avec une variance à long terme $V_{L}=\boldsymbol{\omega} / \gamma$.

Pour analyser l'impact de la crise de Covid, nous procédons à une modélisation en deux périodes :

- une $1^{\text {ère }}$ modélisation avant l'arrivée de la crise de Covid sur la base des données du $\underline{\mathbf{5 / 7 / 2 0 1 0} \text { à }}$ $\underline{31 / 12 / 2019}$.

- une $2^{\text {ème }}$ modélisation après Covid $\underline{5 / 7 / 2021}$ à 10/05/2021.

Les résultats obtenus sur Eviews 12 sont résumés sur le tableau suivant :

\begin{tabular}{|c|c|c|c|c|c|c|}
\hline INDICE & \multicolumn{2}{|l|}{ MASI } & \multicolumn{2}{|l|}{ FTSE 15} & \multicolumn{2}{|c|}{ FTSE ALL } \\
\hline Période & AVANT & APRES & AVANT & APRES & AVANT & APRES \\
\hline$\omega(*)$ & $6.82 \mathrm{E}-06$ & $4.88 \mathrm{E}-06$ & $6.35 \mathrm{E}-06$ & $6.10 \mathrm{E}-06$ & $5.97 \mathrm{E}-06$ & $5.47 \mathrm{E}-06$ \\
\hline$\alpha(*)$ & 0.206997 & 0.196641 & 0.191737 & 0.164953 & 0.174598 & 0.183538 \\
\hline$\beta\left(^{*}\right)$ & 0.598933 & 0.681802 & 0.688198 & 0.706668 & 0.689006 & 0.705344 \\
\hline $\begin{array}{ll}\text { Coefficient } & \text { de } \\
\text { persistance }(\alpha+\beta) & \end{array}$ & 0,805 & 0,878 & 0,871 & 0,88 & 0,863 & 0,888 \\
\hline$y=1-(\alpha+\beta)$ & 0,195 & 0,122 & 0,129 & 0,12 & 0,137 & 0,112 \\
\hline $\begin{array}{l}\text { Volatilité annualisée } \\
2 \sqrt{ } V_{L} * \sqrt{250}\end{array}$ & $9,37 \%$ & $10,02 \%$ & $10,90 \%$ & $11,50 \%$ & $10,46 \%$ & $11,09 \%$ \\
\hline
\end{tabular}

Tableau (3) : Résultat de la modélisation GARCH (1,1) pour les indices MASI, FTSE ALL et FSTE 15.

*) tous les coefficients sont statistiquement non nuls puisque la P-value $<5 \%$

\section{Source : Auteur.}

Dans ce modèle GARCH $(1,1)$, la persistance est mesurée par $(\boldsymbol{\alpha}+\boldsymbol{\beta})$. A partir de données hebdomadaires sur les 3 indices, la persistance de la volatilité des 2 indices FTSE (ALL \& 15) est supérieure à celle de l'indice général de l'ensemble des titres cotés (MASI). Par contre concernant l'impact de la crise, la volatilité générale du marché a augmenté de manière significative contrairement à l'indice FTSE 15 et FTSE ALL LIQUID dont les variations ont légèrement impactées par la pandémie. Cette différence nous permet de dire que les grandes capitalisation, et qui sont en principe les plus liquides, n'ont pas été impactées sensiblement par rapport aux capitalisation moyennes ou faibles. 
Avec un coefficient de persistance aux alentours de $88 \%$ pour les 3 indices indique qu'un retour à la normale en tendant vers la volatilité non conditionnelle $\left(\boldsymbol{V}_{\boldsymbol{L}}\right)$ nécessite du temps en prenant en considération les facteurs éventuels qui permettent la persistance de cette situation.

\section{3-2 Analyse de l'impact de la crise covid et effet d'asymétrie}

Pour vérifier l'effet d'asymétrie sur la volatilité nous allons se limiter à l'utilisation des modèles EGARCH et GJR GARCH à partir du logiciel Eviews 12 en distinguant entre deux périodes : l'avant et l'après Covid.

\section{3-2-2 Modélisation EGARCH}

Il faut signaler qu'il y a une différence entre la spécification EVIEWS du modèle EGARCH qui donne une spécification en logarithme de la variance conditionnelle et le modèle original de Nelson qui donne une spécification en en logarithme de la volatilité conditionnelle.

$$
\log \left(h_{t}\right)=\varphi+\quad \eta_{i}\left|\frac{u_{t-i}}{\sqrt{h_{t-i}}}\right|+\quad \lambda_{i} \frac{u_{t-i}}{\sqrt{h_{t-i}}}+\quad \theta_{k} \log \left(h_{t-k}\right)
$$

Les résultats obtenus sur Eviews 12. sont résumés sur le tableau suivant :

Tableau (4) : Résultat de la modélisation Egarch pour les indices MASI, FTSE ALL et FTSE 15.

\begin{tabular}{|c|c|c|c|c|c|c|}
\hline Indice & \multicolumn{2}{|c|}{ MASI } & \multicolumn{2}{|c|}{ FTSE 15} & \multicolumn{2}{|c|}{ FTSE ALL } \\
\hline Période & AVANT & APRES & AVANT & APRES & AVANT & APRES \\
\hline$\omega$ & $-2.157830(*)$ & $-1.099076\left(^{*}\right)$ & $-1.485413(*)$ & $-1.128182\left(^{*}\right)$ & $-1.435279\left(^{*}\right)$ & $-1.016601(*)$ \\
\hline$\alpha$ & $\left.0.3459322^{*}\right)$ & $0.306307\left(^{*}\right)$ & $0.297774\left(^{*}\right)$ & $0.313657\left(^{*}\right)$ & $0.301114\left(^{*}\right)$ & $0.295411(*)$ \\
\hline$\gamma$ & $-0.005782(* *)$ & $-0.027781\left(^{*}\right)$ & $0.007346\left(^{* *}\right)$ & $-0.017859(* * *)$ & $-0.006464(* *)$ & $-0.022408\left(^{*}\right)$ \\
\hline$B$ & $0.817316\left(^{*}\right)$ & $0.915510\left(^{*}\right)$ & $0.874956\left(^{*}\right)$ & $0.910868\left(^{*}\right)$ & $0.881036\left(^{*}\right)$ & $0.920990\left({ }^{*}\right)$ \\
\hline
\end{tabular}

(*) coefficient statistiquement significatif $(P$-value $<1 \%)$

$(* *)$ coefficient statistiquement non significatif $(P$-value $>10 \%)$

(***) coefficient statistiquement significatif $(P$-value $<10 \%)$

L'examen de la signification statistique des différents coefficients du modèle nous montre clairement qu'avant la période Covid, le comportement de la volatilité ne montre aucun effet de levier d'un point de vue statistique. Pour les 3 indices, le coefficient $\boldsymbol{\gamma}$ est statiquement nul puisque la probabilité de l'hypothèse de nullité est supérieure à 10\%. Mais, depuis l'avènement de la crise du Covid nous assistons un effet de levier significatif très prononcé pour l'indice MASI et donc pour l'ensemble du marché puisque la signification statistique du coefficient $(\boldsymbol{\gamma})$ est non nul au seuil de 1\%. Pour les deux autres indices FSTE 15 et FTSE ALL LIQUID, cette signification statistique est non nulle respectivement au seuil de $10 \%$ et $5 \%$. 
Le coefficient $\boldsymbol{\gamma}<\mathbf{0}$ pour les 3 indices, ce qui indique que pour les 3 indices, les mauvaises nouvelles ont un effet plus important sur la volatilité du titre que les bonnes nouvelles pour les 3 indices depuis l'avènement de la crise.

Tableau (5) : Calcul de l'effet de levier total sur le $\operatorname{Ln}\left(\sigma^{2}\right)$ est :

\begin{tabular}{|l|l|l|l|}
\hline Indice & MASI & FTSE 15 & FTSE ALL \\
\hline Si $\boldsymbol{\varepsilon}_{\mathrm{t}-1}>\mathbf{0}$ alors l'effet total est de $(\boldsymbol{\alpha}+\boldsymbol{\gamma})$ & 0,278526 & 0,295798 & 0,273003 \\
\hline Si $\boldsymbol{\varepsilon}_{\mathrm{t}-1}<\mathbf{0}$, alors l'effet total est de $(\boldsymbol{\alpha} \boldsymbol{\gamma})$ & 0,334088 & 0,331516 & 0,317819 \\
\hline
\end{tabular}

L'effet total est très élevé pour l'ensemble du marché boursier marocain. Par contre moins fort pour les titres les plus liquides que représente l'indice FTSE ALL.

\section{3-2-3. Modélisation GJR GARCH}

Les résultats de cette modélisation obtenus sur Eviews 12 sont résumés sur le tableau suivant :

Tableau (6) : Résultat de la modélisation GJR GARCH pour les indices MASI, FTSE ALL et FSTE 15.

\begin{tabular}{|c|c|c|c|c|c|c|}
\hline$I N D I C E$ & \multicolumn{2}{|c|}{ MASI } & \multicolumn{2}{|c|}{ FTSE 15} & \multicolumn{2}{|c|}{ FTSE ALL } \\
\hline Période & AVANT & APRES & AVANT & APRES & AVANT & APRES \\
\hline C & $6.19 \mathrm{E}-06(*)$ & $4.38 \mathrm{E}-06(*)$ & $5.58 \mathrm{E}-06\left({ }^{*}\right)$ & $5.94 \mathrm{E}-06\left({ }^{*}\right)$ & $5.40 \mathrm{E}-06(*)$ & $5.00 \mathrm{E}-06(*)$ \\
\hline RESID $(-1)^{2}$ & $0.172791\left(^{*}\right)$ & $0.153066(*)$ & $0.151534\left(^{*}\right)$ & $0.165642\left(^{*}\right)$ & $0.145227\left(^{*}\right)$ & $0.147374(*)$ \\
\hline $\begin{array}{l}\text { RESID }(-1)^{2 *}(\text { RESID(- } \\
1)<0)\end{array}$ & $0.024004(* *)$ & $0.041808(*)$ & $0.000422(* *)$ & $0.025282(* * *)$ & $0.028694\left({ }^{* *}\right)$ & $0.041046\left(^{*}\right)$ \\
\hline GARCH(-1) & $0.634478(*)$ & $0.712881\left(^{*}\right)$ & $0.729485(*)$ & $0.708066\left(^{*}\right)$ & $0.715355\left(^{*}\right)$ & $0.728171(*)$ \\
\hline
\end{tabular}

(*) coefficient statistiquement significatif ( $P$-value $<5 \%)$

$(* *)$ coefficient statistiquement non significatif $(P$-value $>5 \%)$

$(* * *)$ coefficient statistiquement non significatif $(P$-value $>10 \%)$

Les résultats révèlent qu'avant la période de Covid, l'effet d'asymétrie en cas d'un choc négatif est statistiquement non significatif ( $p$-value $>\mathbf{5} \%$. Par contre depuis la crise du Covid nous constatons un effet d'asymétrie très prononcé pour les chocs négatifs pour les indices MASI et FTSE ALL. Pour les grandes capitalisations aucun effet d'asymétrie en cas d'un choc négatif ne peut être confirmé.

L'asymétrie peut être visualisée par la courbe d'impact (new impact curve) pour les deux modélisations Egarch et GJR Garch : 

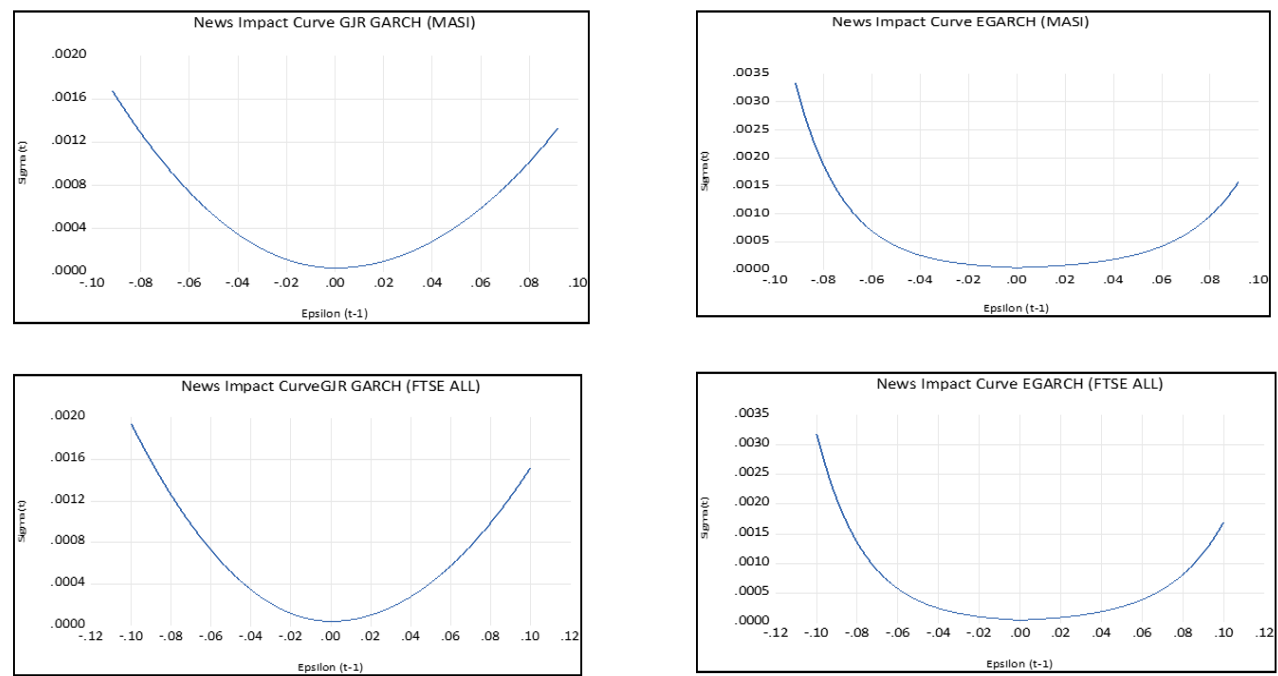

Figure (3) : la courbe d'impact des indices FSTE ALL et MASI par les modèles Egarch et GJR GARCH.

Le choix de la modélisation parfaite dépend de la comparaison des critères d'information comme le résume le tableau suivant :

Tableau (6) : Comparaison des critères pour le choix du modèle optimal.

\begin{tabular}{|l|l|l|l|l|}
\hline INDICE & \multicolumn{2}{|c|}{ MASI } & \multicolumn{2}{c|}{ FTSE ALL } \\
\hline Modèle & EGARCH & GJR GARCH & EGARCH & GJR GARCH \\
\hline $\begin{array}{l}\text { Akaike } \\
\text { criterion }\end{array}$ & -7.469585 & -7.478933 & -7.261497 & -7.268013 \\
\hline Schwarz criterion & -7.458660 & -7.468008 & -7.248555 & -7.255070 \\
\hline $\begin{array}{l}\text { Hannan-Quinn } \\
\text { criter. }\end{array}$ & -7.465634 & -7.474982 & -7.256821 & -7.263336 \\
\hline
\end{tabular}

En définitif, on doit opter pour le modèle qui minimise les critères de sélection. Il est clair que le modèle GJR Garch est le mieux adapté pour décrire et modéliser des effets d'asymétrie sur la volatilité conditionnelle pour les indices MASI et FTSE ALL. Par contre pour l'indice FTSE 15 aucun effet d'asymétrie n'a été vérifiée par la modélisation GJR GARCH.

\section{CONCLUSION}

L'analyse de la volatilité conditionnelle, avant et après la pandémie, du marché boursier marocain a révélé :

- D'une part l'accentuation de la persistance de la volatilité du marché de son ensemble que représente l'indice MASI. Par contre cette persistance n'a pas connu une augmentation très significative pour les titres les plus liquides ou des grandes capitalisations représentés respectivement par les indices FTSE ALL et FTSE 15. Ceci ne permet de déduire que seules les capitalisations moyennes ou faibles et moins liquides ont été sujettes à des volatilités excessives et persistantes à la fois. 
- D'autres part la crise de Covid a donné de l'ampleur et de significativité statistique à l'effet d'asymétrie dû à des chocs négatifs par rapport aux chocs positifs et dont le modèle GJR est le plus adapté pour son modélisation. Il faut signaler que cette asymétrie n'a pas vérifiée pour l'indice des grandes capitalisations.

Les implications des conséquences de la persistance de la volatilité et de l'asymétrie sont d'une grande utilité en matière de gestion d'allocations des portefeuilles pour mieux appréhender le risque des actifs et améliorer la gestion des risques.

\section{BIBLIOGRAPHIE}

[1] Bourse des Valeurs de Casablanca (2021). «Statistiques annuelles de 2020», Publications de la Bourse des Valeurs de Casablanca, Casablanca.

[2] M.Beraich \& M. Amine Fadali, Impact of the covid-19 crisis on the Moroccan stock market: Modeling the volatility of the M.A.S.I stock market index. IJAFAME.Volume 2, Issue 1 (January, 2021), pp. 100-108.

[3] Bollerslev, T. (1986). Generalized autoregressive conditional heteroskedasticity. Journal of Econometrics 31, 307-327.

[4] CHAUDHARY, R., BAKHSHI, P., \& GUPTA, H. (2020a), «Volatility in International Stock Markets: An Empirical Study during COVID-19», Journal of Economic Studies, vol. 13, n9, pp. 208-225.

[5] CDG Capital (2020). «Rapport semestriel boursier du 30 juin 2020 : L'impact Covid-19 pèse lourdement sur la BVC au S1-2020», Publications de CDG Capital, Rabat.

[6] Christophe Hurlin. Econométrie pour la Finance Modèles ARCH - GARCH. Documents et supports de cours 2006-2007. Faculté de Droit, d'Economie et de Gestion.

[7] Engle, R. F \& Bollerslev, T. (1988). Modeling the Persistence of Conditional Variance.Econometric Review. Vol. 5, pp. $1-50$.

[8] Engle, R.F. (1982). Autoregressive Conditional Heteroscedasticity with Estimates of the Variance of United Kingdom Inflation. Econometrica, 50(4), 987-1007.

[9] R. Shiller, « Do Stock Prices Move too Much to be Justifi ed by Subsequent Changes in Dividends », American Economic Review, 71, 3, 1981, p. 421-436.

[10] Sanvi Avouyi-Dovi et Eric Jondeau. La modalisation de la volatilité des bourses asiatiques. Banque de France-Direction générale des études. Janvier 1999.

[11] Zakoian, J. M. (1994). Threshold Heteroskedastic Models, Journal of Economic Dynamics and Control, vol. 18, no. 5, pp. 931-955.

[12] Données numériques téléchargeables sur les sites de la bourse de Casablanca et investing.com. 